\title{
Miscellaneous Graph Preliminaries
}

\author{
Sebastian Koch \\ Johannes Gutenberg University \\ Mainz, Germany ${ }^{1}$
}

Summary. This article contains many auxiliary theorems which were missing in the Mizar Mathematical Library [2] to the best of the author's knowledge. Most of them regard graph theory as formalized in the GLIB series (cf. [8]) and most of them are preliminaries needed in [7] or other forthcoming articles.

MSC: $05 \mathrm{C} 07 \quad 68 \mathrm{~V} 20$

Keywords: graph theory; vertex degrees

MML identifier: GLIBPRE0, version: 8.1.09 5.60.1371

\section{INTRODUCTION}

A generalized approach to graph theory as it was done in [3, 5] in contrast to [9], 4] is rather uncommon. To avoid duplication of the same theorems in different formalization frameworks in the Mizar Mathematical Library [1], a generalized approach to formalization is preferable (cf. [8], 6]). However, due to the sheer amount of "obvious facts" such an approach brings with it, it is only natural some of them not immediately needed slip the initial formalization process. This article aims to fill some of the gaps that emerged. Thereby, in most cases, preliminaries needed in [7] are provided.

Many theorems in this article regard the change of incident edge sets and degrees of a vertex when going from one graph to a related one (e.g. when reversing edge directions or adding an edge).

\footnotetext{
${ }^{1}$ The author is enrolled in the Johannes Gutenberg University in Mayence, Germany, mailto: skoch02@students.uni-mainz.de 


\section{Preliminaries not Directly Related to Graphs}

Let us consider sets $X, Y, Z$. Now we state the propositions:

(1) If $Z \subseteq X$, then $X \cup Y \backslash Z=X \cup Y$.

(2) $X \cap Z$ misses $Y \backslash Z$.

(3) Let us consider objects $x, y$. Then $\{x, y\} \backslash\{$ the element of $\{x, y\}\}=\emptyset$ if and only if $x=y$.

Let us consider objects $a, b, x, y$. Now we state the propositions:

(4) Suppose $a \neq b$ and $x=$ the element of $\{a, b\}$ and $y=$ the element of $\{a, b\} \backslash\{$ the element of $\{a, b\}\}$. Then

(i) $x=a$ and $y=b$, or

(ii) $x=b$ and $y=a$.

(5) $\quad\{a, b\}=\{x, y\}$ if and only if $x=a$ and $y=b$ or $x=b$ and $y=a$.

(6) Let us consider a set $X$, and a non empty set $Y$. Then $X \subset Y$ if and only if $X$ is a proper subset of $Y$.

Let $X$ be a non empty set. One can check that $\operatorname{id}_{X}$ is non irreflexive and $X \times X$ is non irreflexive and non asymmetric and there exists a binary relation on $X$ which is non irreflexive and non asymmetric and there exists a binary relation on $X$ which is symmetric, irreflexive, and non total and there exists a binary relation on $X$ which is symmetric, non irreflexive, and non empty.

Let $X$ be a non trivial set. Observe that $\operatorname{id}_{X}$ is non connected and there exists a binary relation on $X$ which is symmetric and non connected and $X \times X$ is non antisymmetric and there exists a binary relation on $X$ which is non antisymmetric.

Now we state the propositions:

(7) Let us consider binary relations $R, S$, and a set $X$. Then $(R \cup S)^{\circ} X=$ $R^{\circ} X \cup S^{\circ} X$.

(8) Let us consider binary relations $R, S$, and a set $Y$. Then $(R \cup S)^{-1}(Y)=$ $R^{-1}(Y) \cup S^{-1}(Y)$.

(9) Let us consider a binary relation $R$, and sets $X, Y$. Then $(Y \mid R) \uparrow X=$ $(Y \nmid R) \cap(R \uparrow X)$.

(10) Let us consider a symmetric binary relation $R$, and an object $x$. Then $R^{\circ} x=\operatorname{Coim}(R, x)$.

(11) Let us consider a set $X$, and a binary relation $R$ on $X$. Then $R$ is irreflexive if and only if $\mathrm{id}_{X}$ misses $R$.

(12) Let us consider objects $x, y$. Then $(\{\langle x, y\rangle\} \text { qua binary relation })^{\smile}=\{\langle y$, $x\rangle\}$. 
(13) Let us consider a set $X$, objects $x, y$, and a symmetric binary relation $R$ on $X$. If $\langle x, y\rangle \in R$, then $\langle y, x\rangle \in R$.

Let $a, b$ be cardinal numbers. Note that $a \cap b$ is cardinal and $a \cup b$ is cardinal. Let $X$ be a $\subseteq$-linear set. One can check that $\subseteq_{X}$ is connected and $\langle X, \subseteq\rangle$ is connected.

Now we state the propositions:

(14) Let us consider a non empty set $X$. Suppose for every set $a$ such that $a \in X$ holds $a$ is a cardinal number. Then there exists a cardinal number $A$ such that

(i) $A \in X$, and

(ii) $A=\bigcap X$.

Proof: Define $\mathcal{P}$ [ordinal number] $\equiv \$_{1} \in X$ and $\$_{1}$ is a cardinal number. There exists an ordinal number $A$ such that $\mathcal{P}[A]$. Consider $A$ being an ordinal number such that $\mathcal{P}[A]$ and for every ordinal number $B$ such that $\mathcal{P}[B]$ holds $A \subseteq B$.

(15) Let us consider a set $X$. Suppose for every set $a$ such that $a \in X$ holds $a$ is a cardinal number. Then $\bigcap X$ is a cardinal number. The theorem is a consequence of (14).

Let $f$ be a cardinal yielding function and $x$ be an object. Note that $f(x)$ is cardinal.

Let $X$ be a functional set. Note that $\bigcap X$ is function-like and relation-like.

Now we state the propositions:

(16) Let us consider a set $X$. Then $4 \subseteq \overline{\bar{X}}$ if and only if there exist objects $w, x, y, z$ such that $w, x, y, z \in X$ and $w \neq x$ and $w \neq y$ and $w \neq z$ and $x \neq y$ and $x \neq z$ and $y \neq z$.

Proof: If $4 \subseteq \overline{\bar{X}}$, then there exist objects $w, x, y, z$ such that $w, x, y$, $z \in X$ and $w \neq x$ and $w \neq y$ and $w \neq z$ and $x \neq y$ and $x \neq z$ and $y \neq z$.

(17) Let us consider a set $X$. Suppose $4 \subseteq \overline{\bar{X}}$. Let us consider objects $w, x$, $y$. Then there exists an object $z$ such that

(i) $z \in X$, and

(ii) $w \neq z$, and

(iii) $x \neq z$, and

(iv) $y \neq z$.

The theorem is a consequence of (16).

(18) Let us consider a set $X$. Then $S_{X}$ misses 2 Set $X$. 
(19) Let us consider sets $X, Y$. Suppose $\overline{\bar{X}}=\overline{\bar{Y}}$. Then $\overline{\overline{2 \operatorname{Set} X}}=\overline{\overline{2 \operatorname{Set} Y}}$.

Proof: Consider $g$ being a function such that $g$ is one-to-one and dom $g=$ $X$ and $\operatorname{rng} g=Y$. Define $\mathcal{K}($ set $)=$ the element of $\$_{1}$. Define $\mathcal{L}$ (set) $=$ the element of $\$_{1} \backslash\left\{\mathcal{K}\left(\$_{1}\right)\right\}$. Define $\mathcal{F}$ (object) $=\left\{g\left(\mathcal{K}\left(\$_{1}\left(\in 2^{X}\right)\right)\right), g\left(\mathcal{L}\left(\$_{1}(\in\right.\right.\right.$ $\left.\left.2^{X}\right)\right)$ ) . Consider $f$ being a function such that $\operatorname{dom} f=2 \operatorname{Set} X$ and for every object $x$ such that $x \in 2 \operatorname{Set} X$ holds $f(x)=\mathcal{F}(x)$.

(20) Let us consider a finite set $X$. Then $\overline{\overline{2 \operatorname{Set} X}}=\left(\begin{array}{c}\overline{\bar{X}} \\ 2\end{array}\right)$. The theorem is a consequence of (19).

\section{InTO GLIB_000}

Now we state the propositions:

(21) Let us consider a graph $G$, a vertex $v$ of $G$, and objects $e, w$. If $v$ is isolated, then $e$ does not join $v$ and $w$ in $G$.

(22) Let us consider a graph $G$, a vertex $v$ of $G$, and objects $e, w$. Suppose $v$ is isolated. Then

(i) $e$ does not join $v$ to $w$ in $G$, and

(ii) $e$ does not join $w$ to $v$ in $G$.

The theorem is a consequence of (21).

(23) Let us consider a graph $G$, and a vertex $v$ of $G$. Then $v$ is isolated if and only if $v \notin \operatorname{rng}$ (the source of $G$ ) $\cup \operatorname{rng}$ (the target of $G$ ). The theorem is a consequence of $(22)$.

(24) Let us consider a graph $G$, a vertex $v$ of $G$, and an object $e$. If $v$ is endvertex, then $e$ does not join $v$ and $v$ in $G$.

(25) Let us consider a graph $G$, and a vertex $v$ of $G$. Then

(i) $v \cdot \operatorname{edges} \operatorname{In}()=(\text { the target of } G)^{-1}(\{v\})$, and

(ii) $v$.edgesOut ()$=(\text { the source of } G)^{-1}(\{v\})$.

Let us consider a trivial graph $G$ and a vertex $v$ of $G$. Now we state the propositions:

(26)

(i) $v$.edges $\operatorname{In}()=$ the edges of $G$, and

(ii) $v$.edgesOut ()$=$ the edges of $G$, and

(iii) $v$.edgesInOut ()$=$ the edges of $G$.

(i) $v$.inDegree ()$=G$.size () , and

(ii) $v$.outDegree ()$=G$.size () , and 
(iii) $v \cdot \operatorname{degree}()=G \cdot \operatorname{size}()+G \cdot \operatorname{size}()$.

The theorem is a consequence of (26).

(28) Let us consider a graph $G$, and sets $X, Y$. Then $G$.edgesBetween $(X, Y)=$ $G$.edgesDBetween $(X, Y) \cup G$.edgesDBetween $(Y, X)$.

(29) Let us consider a graph $G$, and a vertex $v$ of $G$. Then $v$.edgesInOut ()$=$ $G$.edgesBetween(the vertices of $G,\{v\})$. The theorem is a consequence of (28).

Let us consider a graph $G$ and sets $X, Y$. Now we state the propositions:

(30) $G$.edgesDBetween $(X, Y)=G$.edgesOutOf $(X) \cap G$.edgesInto $(Y)$.

(31) $G$.edgesDBetween $(X, Y) \subseteq G$.edgesBetween $(X, Y)$.

Let us consider a graph $G$ and a vertex $v$ of $G$. Now we state the propositions:

(32) If for every object $e, e$ does not join $v$ and $v$ in $G$, then $\overline{\overline{v \text {.edgesInOut() }}}=$ $v$.degree () .

Proof: $v$.edgesIn ()$\cap v$.edgesOut ()$=\emptyset$.

(33) $\quad v$ is isolated if and only if $v \cdot \operatorname{edges} \operatorname{In}()=\emptyset$ and $v \cdot \operatorname{edgesOut}()=\emptyset$.

(34) $v$ is isolated if and only if $v$.inDegree ()$=0$ and $v$.outDegree ()$=0$. The theorem is a consequence of (33).

(35) $v$ is isolated if and only if $v \cdot \operatorname{degree}()=0$. The theorem is a consequence of $(34)$.

Let us consider a graph $G$ and a set $X$. Now we state the propositions:

(36) $G$.edgesInto $(X)=\bigcup\{v$.edgesIn(), where $v$ is a vertex of $G: v \in X\}$.

(37) $G$.edgesOutOf $(X)=\bigcup\{v$.edgesOut () , where $v$ is a vertex of $G: v \in$ $X\}$.

(38) G.edgesInOut $(X)=\bigcup\{v$.edgesInOut(), where $v$ is a vertex of $G: v \in$ $X\}$.

Let us consider a graph $G$ and sets $X, Y$. Now we state the propositions:

(39) $G$.edgesDBetween $(X, Y)=\bigcup\{v$.edgesOut ()$\cap w$.edgesIn( () , where $v, w$ are vertices of $G: v \in X$ and $w \in Y\}$.

(40) G.edgesBetween $(X, Y) \subseteq \bigcup\{v$.edgesInOut ()$\cap w$. edgesInOut () , where $v, w$ are vertices of $G: v \in X$ and $w \in Y\}$.

(41) Suppose $X$ misses $Y$. Then $G$.edgesBetween $(X, Y)=\bigcup\{v$.edgesInOut ()$\cap$ $w$.edgesInOut(), where $v, w$ are vertices of $G: v \in X$ and $w \in Y\}$. The theorem is a consequence of (40).

(42) Let us consider a graph $G_{1}$, a set $E$, a subgraph $G_{2}$ of $G_{1}$ with edges $E$ removed, a vertex $v_{1}$ of $G_{1}$, and a vertex $v_{2}$ of $G_{2}$. Suppose $v_{1}=v_{2}$. Then

(i) $v_{2} \cdot \operatorname{edg} \operatorname{es} \operatorname{In}()=v_{1} \cdot$ edges $\operatorname{In}() \backslash E$, and

(ii) $v_{2}$.edgesOut ()$=v_{1}$.edgesOut ()$\backslash E$, and 
(iii) $v_{2}$.edgesInOut ()$=v_{1}$.edgesInOut ()$\backslash E$.

(43) Let us consider graphs $G_{1}, G_{2}$, and a set $V$. Then $G_{2}$ is a subgraph of $G_{1}$ with vertices $V$ removed if and only if $G_{2}$ is a subgraph of $G_{1}$ with vertices $V \cap$ (the vertices of $G_{1}$ ) removed.

(44) Let us consider a graph $G_{1}$, a set $V$, a subgraph $G_{2}$ of $G_{1}$ with vertices $V$ removed, a vertex $v_{1}$ of $G_{1}$, and a vertex $v_{2}$ of $G_{2}$. Suppose $V \subset$ the vertices of $G_{1}$ and $v_{1}=v_{2}$. Then

(i) $v_{2}$.edges $\operatorname{In}()=v_{1}$.edgesIn ()$\backslash\left(G_{1}\right.$.edgesOutOf $\left.(V)\right)$, and

(ii) $v_{2}$.edgesOut ()$=v_{1}$.edgesOut ()$\backslash\left(G_{1}\right.$.edgesInto $\left.(V)\right)$, and

(iii) $v_{2}$.edgesInOut ()$=v_{1}$.edgesInOut ()$\backslash\left(G_{1}\right.$.edgesInOut $\left.(V)\right)$.

Proof: $v_{1}$.edgesOut ()$\cap G_{1}$.edgesOutOf $(V)=\emptyset$. $v_{1}$.edgesIn ()$\cap G_{1}$.edgesInto $(V)=\emptyset$.

(45) Let us consider a non trivial graph $G_{1}$, a vertex $v$ of $G_{1}$, a subgraph $G_{2}$ of $G_{1}$ with vertex $v$ removed, a vertex $v_{1}$ of $G_{1}$, and a vertex $v_{2}$ of $G_{2}$. Suppose $v_{1}=v_{2}$. Then

(i) $v_{2} \cdot \operatorname{edges} \operatorname{In}()=v_{1} \cdot \operatorname{edges} \operatorname{In}() \backslash(v \cdot \operatorname{edgesOut}())$, and

(ii) $v_{2}$.edgesOut ()$=v_{1}$.edgesOut ()$\backslash(v$.edgesIn ()$)$, and

(iii) $v_{2}$.edgesInOut ()$=v_{1}$.edgesInOut ()$\backslash(v$.edgesInOut ()$)$.

The theorem is a consequence of (44).

\section{INTO GLIB_002}

Now we state the proposition:

(46) Let us consider a graph $G$, a component $C$ of $G$, a vertex $v_{1}$ of $G$, and a vertex $v_{2}$ of $C$. Suppose $v_{1}=v_{2}$. Then

(i) $v_{1} \cdot \operatorname{edges} \operatorname{In}()=v_{2} \cdot \operatorname{edges} \operatorname{In}()$, and

(ii) $v_{1}$.inDegree ()$=v_{2}$.inDegree () , and

(iii) $v_{1}$.edgesOut ()$=v_{2}$.edgesOut () , and

(iv) $v_{1}$.outDegree ()$=v_{2}$.outDegree $($ ), and

(v) $v_{1}$.edgesInOut ()$=v_{2}$.edgesInOut () , and

(vi) $v_{1}$.degree ()$=v_{2} \cdot \operatorname{degree}()$. 


\section{InTo GLIB_006}

Now we state the propositions:

(47) Let us consider a graph $G_{2}$, a set $V$, a supergraph $G_{1}$ of $G_{2}$ extended by the vertices from $V$, a vertex $v_{1}$ of $G_{1}$, and a vertex $v_{2}$ of $G_{2}$. Suppose $v_{1}=v_{2}$. Then

(i) $v_{1} \cdot \operatorname{edg} \operatorname{es} \operatorname{In}()=v_{2} \cdot \operatorname{edges} \operatorname{In}()$, and

(ii) $v_{1}$.inDegree ()$=v_{2}$.inDegree () , and

(iii) $v_{1}$.edgesOut ()$=v_{2}$.edgesOut () , and

(iv) $v_{1}$.outDegree ()$=v_{2}$.outDegree () , and

(v) $v_{1}$.edgesInOut ()$=v_{2}$.edgesInOut () , and

(vi) $v_{1} \cdot \operatorname{degree}()=v_{2} \cdot \operatorname{degree}()$.

(48) Let us consider a graph $G_{2}$, objects $v, w$, e, a supergraph $G_{1}$ of $G_{2}$ extended by $e$ between vertices $v$ and $w$, a vertex $v_{1}$ of $G_{1}$, and a vertex $v_{2}$ of $G_{2}$. Suppose $v_{1}=v_{2}$ and $v_{2} \neq v$ and $v_{2} \neq w$. Then

(i) $v_{1} \cdot \operatorname{edg} \operatorname{es} \operatorname{In}()=v_{2} \cdot \operatorname{edg} \operatorname{es} \operatorname{In}()$, and

(ii) $v_{1}$.inDegree ()$=v_{2}$.inDegree () , and

(iii) $v_{1}$.edgesOut ()$=v_{2}$.edgesOut () , and

(iv) $v_{1}$.outDegree ()$=v_{2}$.outDegree () , and

(v) $v_{1}$.edgesInOut ()$=v_{2}$.edgesInOut () , and

(vi) $v_{1} \cdot \operatorname{degree}()=v_{2} \cdot \operatorname{degree}()$.

(49) Let us consider a graph $G_{2}$, vertices $v, w$ of $G_{2}$, an object $e$, a supergraph $G_{1}$ of $G_{2}$ extended by $e$ between vertices $v$ and $w$, and a vertex $v_{1}$ of $G_{1}$. Suppose $e \notin$ the edges of $G_{2}$ and $v_{1}=v$ and $v \neq w$. Then

(i) $v_{1} \cdot$ edges $\operatorname{In}()=v \cdot$ edges $\operatorname{In}()$, and

(ii) $v_{1} \cdot \operatorname{inDegree}()=v \cdot$ inDegree () , and

(iii) $v_{1}$.edgesOut ()$=v$.edgesOut ()$\cup\{e\}$, and

(iv) $v_{1}$.outDegree ()$=v$.outDegree ()$+1$, and

(v) $v_{1}$.edgesInOut ()$=v$.edgesInOut ()$\cup\{e\}$, and

(vi) $v_{1}$.degree ()$=v \cdot \operatorname{degree}()+1$.

(50) Let us consider a graph $G_{2}$, vertices $v, w$ of $G_{2}$, an object $e$, a supergraph $G_{1}$ of $G_{2}$ extended by $e$ between vertices $v$ and $w$, and a vertex $w_{1}$ of $G_{1}$. Suppose $e \notin$ the edges of $G_{2}$ and $w_{1}=w$ and $v \neq w$. Then

(i) $w_{1} \cdot \operatorname{edges} \operatorname{In}()=w \cdot$ edges $\operatorname{In}() \cup\{e\}$, and 
(ii) $w_{1}$.inDegree ()$=w \cdot$ inDegree ()$+1$, and

(iii) $w_{1}$.edgesOut ()$=w$.edgesOut () , and

(iv) $w_{1}$.outDegree ()$=w$.outDegree () , and

(v) $w_{1}$.edgesInOut ()$=w$.edgesInOut ()$\cup\{e\}$, and

(vi) $w_{1} \cdot \operatorname{degree}()=w \cdot \operatorname{degree}()+1$.

(51) Let us consider a graph $G_{2}$, a vertex $v$ of $G_{2}$, an object $e$, a supergraph $G_{1}$ of $G_{2}$ extended by $e$ between vertices $v$ and $v$, and a vertex $v_{1}$ of $G_{1}$. Suppose $e \notin$ the edges of $G_{2}$ and $v_{1}=v$. Then

(i) $v_{1} \cdot \operatorname{edges} \operatorname{In}()=v$.edges $\operatorname{In}() \cup\{e\}$, and

(ii) $v_{1}$.inDegree ()$=v \cdot$ inDegree ()$+1$, and

(iii) $v_{1}$.edgesOut ()$=v$.edgesOut ()$\cup\{e\}$, and

(iv) $v_{1}$.outDegree ()$=v$.outDegree ()$+1$, and

(v) $v_{1}$.edgesInOut ()$=v$.edgesInOut ()$\cup\{e\}$, and

(vi) $v_{1}$.degree ()$=v \cdot \operatorname{degree}()+2$.

\section{InTO GLIB_007}

Now we state the propositions:

(52) Let us consider a graph $G_{3}$, a set $E$, a graph $G_{4}$ given by reversing directions of the edges $E$ of $G_{3}$, a supergraph $G_{1}$ of $G_{3}$, and a graph $G_{2}$ given by reversing directions of the edges $E$ of $G_{1}$. Suppose $E \subseteq$ the edges of $G_{3}$. Then $G_{2}$ is a supergraph of $G_{4}$.

(53) Let us consider a graph $G_{2}$, and an object $v$. Then every supergraph of $G_{2}$ extended by $v$ is a supergraph of $G_{2}$ extended by vertex $v$ and edges between $v$ and $\emptyset$ of $G_{2}$.

(54) Let us consider a graph $G_{1}$, a set $E$, a graph $G_{2}$ given by reversing directions of the edges $E$ of $G_{1}$, a vertex $v_{1}$ of $G_{1}$, and a vertex $v_{2}$ of $G_{2}$. Suppose $v_{1}=v_{2}$ and $E \subseteq$ the edges of $G_{1}$. Then

(i) $v_{2}$.edgesIn( ()$=v_{1}$.edgesIn( ()$\backslash E \cup v_{1}$.edgesOut ()$\cap E$, and

(ii) $v_{2}$.edgesOut ()$=v_{1}$.edgesOut ()$\backslash E \cup v_{1}$.edgesIn ()$\cap E$.

(55) Let us consider a graph $G_{1}$, a graph $G_{2}$ given by reversing directions of the edges of $G_{1}$, a vertex $v_{1}$ of $G_{1}$, and a vertex $v_{2}$ of $G_{2}$. Suppose $v_{1}=v_{2}$. Then

(i) $v_{2} \cdot \operatorname{edges} \operatorname{In}()=v_{1} \cdot \operatorname{edgesOut}()$, and

(ii) $v_{2}$.inDegree ()$=v_{1}$.outDegree () , and 
(iii) $v_{2}$.edgesOut ()$=v_{1}$.edges $\operatorname{In}()$, and

(iv) $v_{2}$.outDegree ()$=v_{1}$.inDegree $($ ).

(56) Let us consider a graph $G_{1}$, a set $E$, a graph $G_{2}$ given by reversing directions of the edges $E$ of $G_{1}$, a vertex $v_{1}$ of $G_{1}$, and a vertex $v_{2}$ of $G_{2}$. Suppose $v_{1}=v_{2}$. Then

(i) $v_{2}$.edgesInOut ()$=v_{1}$.edgesInOut () , and

(ii) $v_{2}$.degree ()$=v_{1}$.degree () .

The theorem is a consequence of (54) and (2).

(57) Let us consider a graph $G_{2}$, an object $v$, a set $V$, a supergraph $G_{1}$ of $G_{2}$ extended by vertex $v$ and edges between $v$ and $V$ of $G_{2}$, and a vertex $w$ of $G_{1}$. Suppose $V \subseteq$ the vertices of $G_{2}$ and $v \notin$ the vertices of $G_{2}$ and $v=w$. Then

(i) $w$.allNeighbors ()$=V$, and

(ii) $w \cdot \operatorname{degree}()=\overline{\bar{V}}$.

The theorem is a consequence of (29), (32), and (35).

(58) Let us consider a graph $G_{2}$, an object $v$, a set $V$, a supergraph $G_{1}$ of $G_{2}$ extended by vertex $v$ and edges between $v$ and $V$ of $G_{2}$, a vertex $v_{1}$ of $G_{1}$, and a vertex $v_{2}$ of $G_{2}$. Suppose $v_{1}=v_{2}$ and $v_{2} \notin V$. Then

(i) $v_{1} \cdot$ edges $\operatorname{In}()=v_{2}$.edgesIn () , and

(ii) $v_{1}$.inDegree ()$=v_{2}$.inDegree () , and

(iii) $v_{1}$.edgesOut ()$=v_{2}$.edgesOut () , and

(iv) $v_{1}$.outDegree ()$=v_{2}$.outDegree () , and

(v) $v_{1}$.edgesInOut ()$=v_{2}$.edgesInOut () , and

(vi) $v_{1}$.degree ()$=v_{2}$.degree () .

(59) Let us consider a graph $G_{2}$, an object $v$, a subset $V$ of the vertices of $G_{2}$, a supergraph $G_{1}$ of $G_{2}$ extended by vertex $v$ and edges between $v$ and $V$ of $G_{2}$, a vertex $v_{1}$ of $G_{1}$, and a vertex $v_{2}$ of $G_{2}$. Suppose $v \notin$ the vertices of $G_{2}$ and $v_{1}=v_{2}$ and $v_{2} \in V$. Then

(i) $v_{1}$.allNeighbors ()$=v_{2}$.allNeighbors ()$\cup\{v\}$, and

(ii) $v_{1} \cdot \operatorname{degree}()=v_{2} \cdot \operatorname{degree}()+1$.

(60) Let us consider a graph $G_{2}$, an object $v$, a set $V$, a supergraph $G_{1}$ of $G_{2}$ extended by vertex $v$ and edges between $v$ and $V$ of $G_{2}$, a vertex $v_{1}$ of $G_{1}$, and a vertex $v_{2}$ of $G_{2}$. Suppose $v_{1}=v_{2}$. Then

(i) $v_{1}$.degree ()$\subseteq v_{2} \cdot$ degree ()$+1$, and 
(ii) $v_{1}$.inDegree ()$\subseteq v_{2}$.inDegree ()$+1$, and

(iii) $v_{1}$.outDegree ()$\subseteq v_{2}$.outDegree ()$+1$.

The theorem is a consequence of (58).

\section{INTO GLIB_008}

Now we state the propositions:

(61) Let us consider a graph $G$. Then $G$ is edgeless if and only if for every vertices $v, w$ of $G, v$ and $w$ are not adjacent.

(62) Let us consider a loopless graph $G$. Then $G$ is edgeless if and only if for every vertices $v, w$ of $G$ such that $v \neq w$ holds $v$ and $w$ are not adjacent. The theorem is a consequence of (61).

\section{INTO GLIB_009}

Now we state the propositions:

(63) Let us consider a graph $G$. Then $G$.loops ()$=\operatorname{dom}(($ the source of $G) \cap$ (the target of $G)$ ).

(64) Let us consider graphs $G_{1}, G_{2}$, and a set $E$. Then $G_{2}$ is a graph given by reversing directions of the edges $E$ of $G_{1}$ if and only if $G_{2}$ is a graph given by reversing directions of the edges $E \backslash\left(G_{1}\right.$.loops ()$)$ of $G_{1}$.

(65) Let us consider a graph $G_{1}$, a subgraph $G_{2}$ of $G_{1}$ with loops removed, a vertex $v_{1}$ of $G_{1}$, and a vertex $v_{2}$ of $G_{2}$. Suppose $v_{1}=v_{2}$. Then

(i) $v_{2}$.inNeighbors ()$=v_{1}$.inNeighbors ()$\backslash\left\{v_{1}\right\}$, and

(ii) $v_{2}$.outNeighbors ()$=v_{1}$.outNeighbors ()$\backslash\left\{v_{1}\right\}$, and

(iii) $v_{2}$.allNeighbors ()$=v_{1}$.allNeighbors ()$\backslash\left\{v_{1}\right\}$.

(66) Let us consider a graph $G_{1}$, a subgraph $G_{2}$ of $G_{1}$ with parallel edges removed, a vertex $v_{1}$ of $G_{1}$, and a vertex $v_{2}$ of $G_{2}$. If $v_{1}=v_{2}$, then $v_{2}$.allNeighbors ()$=v_{1}$.allNeighbors () .

(67) Let us consider a graph $G_{1}$, a subgraph $G_{2}$ of $G_{1}$ with directed-parallel edges removed, a vertex $v_{1}$ of $G_{1}$, and a vertex $v_{2}$ of $G_{2}$. Suppose $v_{1}=v_{2}$. Then

(i) $v_{2}$.inNeighbors ()$=v_{1}$.inNeighbors () , and

(ii) $v_{2}$.outNeighbors ()$=v_{1}$.outNeighbors () , and

(iii) $v_{2}$.allNeighbors ()$=v_{1}$.allNeighbors () . 
(68) Let us consider a graph $G_{1}$, a simple graph $G_{2}$ of $G_{1}$, a vertex $v_{1}$ of $G_{1}$, and a vertex $v_{2}$ of $G_{2}$. Suppose $v_{1}=v_{2}$. Then $v_{2}$.allNeighbors ()$=$ $v_{1}$.allNeighbors ()$\backslash\left\{v_{1}\right\}$. The theorem is a consequence of (65) and (66).

(69) Let us consider a graph $G_{1}$, a directed-simple graph $G_{2}$ of $G_{1}$, a vertex $v_{1}$ of $G_{1}$, and a vertex $v_{2}$ of $G_{2}$. Suppose $v_{1}=v_{2}$. Then

(i) $v_{2}$.inNeighbors ()$=v_{1}$.inNeighbors ()$\backslash\left\{v_{1}\right\}$, and

(ii) $v_{2}$.outNeighbors ()$=v_{1}$.outNeighbors ()$\backslash\left\{v_{1}\right\}$, and

(iii) $v_{2}$.allNeighbors ()$=v_{1}$.allNeighbors ()$\backslash\left\{v_{1}\right\}$.

The theorem is a consequence of (65) and (67).

Let $G$ be a non loopless graph. One can verify that every subgraph of $G$ with parallel edges removed is non loopless and every subgraph of $G$ with directedparallel edges removed is non loopless.

Let $G$ be a non edgeless graph. Note that every subgraph of $G$ with parallel edges removed is non edgeless and every subgraph of $G$ with directed-parallel edges removed is non edgeless.

Now we state the propositions:

(70) Let us consider a graph $G$, and a representative selection of the parallel edges $E$ of $G$. Then $\overline{\bar{E}}=\overline{\overline{\text { Classes EdgeParEqRel }(G)}}$.

Proof: Define $\mathcal{F}$ (object) $=\left[\$_{1}\right]_{\operatorname{EdgeParEqRel}(G)}$. Consider $f$ being a function such that $\operatorname{dom} f=E$ and for every object $x$ such that $x \in E$ holds $f(x)=\mathcal{F}(x)$.

(71) Let us consider a graph $G$, and a representative selection of the directedparallel edges $E$ of $G$. Then $\overline{\bar{E}}=\overline{\overline{\text { Classes DEdgeParEqRel }(G)}}$.

Proof: Define $\mathcal{F}$ (object) $=\left[\$_{1}\right]_{\text {DEdgeParEqRel }(G)}$. Consider $f$ being a function such that $\operatorname{dom} f=E$ and for every object $x$ such that $x \in E$ holds $f(x)=\mathcal{F}(x)$.

(72) Let us consider a graph $G$, a set $X$, a subset $E$ of the edges of $G$, and a graph $H$ given by reversing directions of the edges $X$ of $G$. Then $E$ is a representative selection of the parallel edges of $G$ if and only if $E$ is a representative selection of the parallel edges of $H$.

(73) Let us consider a graph $G$, a non empty subset $V$ of the vertices of $G$, a subgraph $H$ of $G$ induced by $V$, and a representative selection of the parallel edges $E$ of $G$. Then $E \cap G$.edgesBetween $(V)$ is a representative selection of the parallel edges of $H$.

(74) Let us consider a graph $G$, a non empty subset $V$ of the vertices of $G$, a subgraph $H$ of $G$ induced by $V$, and a representative selection of the directed-parallel edges $E$ of $G$. Then $E \cap G$.edgesBetween $(V)$ is a representative selection of the directed-parallel edges of $H$. 
Let us consider a graph $G$, a set $V$, a supergraph $H$ of $G$ extended by the vertices from $V$, and a subset $E$ of the edges of $G$. Now we state the propositions:

(75) $E$ is a representative selection of the parallel edges of $G$ if and only if $E$ is a representative selection of the parallel edges of $H$.

(76) $E$ is a representative selection of the directed-parallel edges of $G$ if and only if $E$ is a representative selection of the directed-parallel edges of $H$.

Note that there exists a graph which is non non-multi and non-directedmulti.

Let $G_{F}$ be a graph-yielding function. We say that $G_{F}$ is plain if and only if

(Def. 1) for every object $x$ such that $x \in \operatorname{dom} G_{F}$ there exists a graph $G$ such that $G_{F}(x)=G$ and $G$ is plain.

Let $G$ be a plain graph. Note that $\langle G\rangle$ is plain and $\mathbb{N} \longmapsto G$ is plain.

Let $G_{F}$ be a non empty, graph-yielding function. One can check that $G_{F}$ is plain if and only if the condition (Def. 2) is satisfied.

(Def. 2) for every element $x$ of $\operatorname{dom} G_{F}, G_{F}(x)$ is plain.

Let $G_{S q}$ be a graph sequence. Note that $G_{S q}$ is plain if and only if the condition (Def. 3) is satisfied.

(Def. 3) for every natural number $n, G_{S q}(n)$ is plain.

Observe that every graph-yielding function which is empty is also plain and there exists a graph sequence which is plain and there exists a graph-yielding finite sequence which is non empty and plain.

Let $G_{F}$ be a plain, non empty, graph-yielding function and $x$ be an element of $\operatorname{dom} G_{F}$. Let us observe that $G_{F}(x)$ is plain. Let $G_{S q}$ be a plain graph sequence and $x$ be a natural number. Let us observe that $G_{S q}(x)$ is plain. Let $p$ be a plain, graph-yielding finite sequence and $n$ be a natural number. One can check that $p \nmid n$ is plain and $p_{\lfloor n}$ is plain. Let $m$ be a natural number.

Observe that $\operatorname{smid}(p, m, n)$ is plain and $\langle p(m), \ldots, p(n)\rangle$ is plain. Let $p, q$ be plain, graph-yielding finite sequences. One can check that $p^{-} q$ is plain and $p \curvearrowright q$ is plain. Let $G_{1}, G_{2}$ be plain graphs. Let us observe that $\left\langle G_{1}, G_{2}\right\rangle$ is plain. Let $G_{3}$ be a plain graph. One can verify that $\left\langle G_{1}, G_{2}, G_{3}\right\rangle$ is plain.

\section{INTO GLIB_010}

Let us consider graphs $G_{1}, G_{2}$. Now we state the propositions:

(77) If $G_{1} \approx G_{2}$, then there exists a partial graph mapping $F$ from $G_{1}$ to $G_{2}$ such that $F=\operatorname{id}_{G_{1}}$ and $F$ is directed-isomorphism.

(78) If $G_{1} \approx G_{2}$, then $G_{2}$ is $G_{1}$-directed-isomorphic. The theorem is a consequence of (77). 
(79) Let us consider a graph $G_{1}$, a set $E$, and a graph $G_{2}$ given by reversing directions of the edges $E$ of $G_{1}$. Then there exists a partial graph mapping $F$ from $G_{1}$ to $G_{2}$ such that

(i) $F=\operatorname{id}_{G_{1}}$, and

(ii) $F$ is isomorphism.

(80) Let us consider a graph $G_{1}$, and a set $E$. Then every graph given by reversing directions of the edges $E$ of $G_{1}$ is $G_{1}$-isomorphic. The theorem is a consequence of (79).

(81) Let us consider graphs $G_{1}, G_{2}$, and a partial graph mapping $F$ from $G_{1}$ to $G_{2}$. Suppose $F$ is directed-continuous and isomorphism. Then

(i) $G_{1}$ is non-directed-multi iff $G_{2}$ is non-directed-multi, and

(ii) $G_{1}$ is directed-simple iff $G_{2}$ is directed-simple.

Let us consider graphs $G_{1}, G_{2}$, a partial graph mapping $F$ from $G_{1}$ to $G_{2}$, and a vertex $v$ of $G_{1}$. Now we state the propositions:

(82) If $v \in \operatorname{dom}\left(F_{\mathbb{V}}\right)$, then $\left(F_{\mathbb{E}}\right)^{\circ}(v$.edgesInOut ()$) \subseteq\left(F_{\mathbb{V}}\right) / v$.edgesInOut () .

(83) Suppose $F$ is directed and $v \in \operatorname{dom}\left(F_{\mathbb{V}}\right)$. Then

(i) $\left(F_{\mathbb{E}}\right)^{\circ}(v \cdot$ edgesIn ()$) \subseteq\left(F_{\mathbb{V}}\right) / v \cdot \operatorname{edges} \operatorname{In}()$, and

(ii) $\left(F_{\mathbb{E}}\right)^{\circ}(v$.edgesOut ()$) \subseteq\left(F_{\mathbb{V}}\right) / v$.edgesOut () .

(84) Suppose $F$ is onto and semi-continuous and $v \in \operatorname{dom}\left(F_{\mathbb{V}}\right)$.

Then $\left(F_{\mathbb{E}}\right)^{\circ}(v$.edgesInOut ()$)=\left(F_{\mathbb{V}}\right) / v$.edgesInOut () . The theorem is a consequence of $(82)$.

(85) Suppose $F$ is onto and semi-directed-continuous and $v \in \operatorname{dom}\left(F_{\mathbb{V}}\right)$. Then

(i) $\left(F_{\mathbb{E}}\right)^{\circ}(v \cdot \operatorname{edges} \operatorname{In}())=\left(F_{\mathbb{V}}\right) / v \cdot \operatorname{edges} \operatorname{In}()$, and

(ii) $\left(F_{\mathbb{E}}\right)^{\circ}(v$.edgesOut ()$)=\left(F_{\mathbb{V}}\right) / v$.edgesOut () .

The theorem is a consequence of (83).

(86) If $F$ is isomorphism, then $\left(F_{\mathbb{E}}\right)^{\circ}(v$.edgesInOut ()$)=\left(F_{\mathbb{V}}\right)_{/ v}$.edgesInOut () . The theorem is a consequence of (84).

(87) Suppose $F$ is directed-isomorphism. Then

(i) $\left(F_{\mathbb{E}}\right)^{\circ}(v$.edgesIn ()$)=\left(F_{\mathbb{V}}\right) / v \cdot \operatorname{edgesIn}()$, and

(ii) $\left(F_{\mathbb{E}}\right)^{\circ}(v$.edgesOut ()$)=\left(F_{\mathbb{V}}\right) / v$.edgesOut () .

The theorem is a consequence of (85).

Let $G_{1}$ be a graph and $G_{2}$ be an edgeless graph. Note that every partial graph mapping from $G_{1}$ to $G_{2}$ is directed.

Let us consider graphs $G_{1}, G_{2}$ and a partial graph mapping $F_{0}$ from $G_{1}$ to $G_{2}$. Now we state the propositions: 
(88) Suppose $F_{0 \mathbb{E}}$ is one-to-one. Then there exists a subset $E$ of the edges of $G_{2}$ such that for every graph $G_{3}$ given by reversing directions of the edges $E$ of $G_{2}$.

There exists a partial graph mapping $F$ from $G_{1}$ to $G_{3}$ such that $F=F_{0}$ and $F$ is directed and if $F_{0}$ is not empty, then $F$ is not empty and if $F_{0}$ is total, then $F$ is total and if $F_{0}$ is one-to-one, then $F$ is oneto-one and if $F_{0}$ is onto, then $F$ is onto and if $F_{0}$ is semi-continuous, then $F$ is semi-continuous and if $F_{0}$ is continuous, then $F$ is continuous. The theorem is a consequence of (79).

(89) Suppose $F_{0 \mathbb{E}}$ is one-to-one. Then there exists a subset $E$ of the edges of $G_{2}$ such that for every graph $G_{3}$ given by reversing directions of the edges $E$ of $G_{2}$.

There exists a partial graph mapping $F$ from $G_{1}$ to $G_{3}$ such that $F=F_{0}$ and $F$ is directed and if $F_{0}$ is weak subgraph embedding, then $F$ is weak subgraph embedding and if $F_{0}$ is strong subgraph embedding, then $F$ is strong subgraph embedding and if $F_{0}$ is isomorphism, then $F$ is isomorphism. The theorem is a consequence of (88).

Let us consider graphs $G_{1}, G_{2}$, a partial graph mapping $F$ from $G_{1}$ to $G_{2}$, and a vertex $v$ of $G_{1}$. Now we state the propositions:

(90) Suppose $F$ is directed and weak subgraph embedding. Then

(i) $v$.inDegree ()$\subseteq\left(F_{\mathbb{V}}\right) / v$.inDegree () , and

(ii) $v$.outDegree ()$\subseteq\left(F_{\mathbb{V}}\right)_{/ v}$.outDegree () .

The theorem is a consequence of (83).

(91) If $F$ is weak subgraph embedding, then $v$.degree ()$\subseteq\left(F_{\mathbb{V}}\right)_{/ v}$.degree () . The theorem is a consequence of (89) and (56).

(92) Suppose $F$ is onto and semi-directed-continuous and $v \in \operatorname{dom}\left(F_{\mathbb{V}}\right)$. Then

(i) $\left(F_{\mathbb{V}}\right)_{/ v}$.inDegree ()$\subseteq v$.inDegree () , and

(ii) $\left(F_{\mathbb{V}}\right)_{/ v}$.outDegree ()$\subseteq v$. outDegree () .

The theorem is a consequence of (85).

(93) If $F$ is onto and semi-directed-continuous and $v \in \operatorname{dom}\left(F_{\mathbb{V}}\right)$, then $\left(F_{\mathbb{V}}\right)_{/ v}$.degree ()$\subseteq v$.degree () . The theorem is a consequence of $(92)$.

(94) If $F$ is directed-isomorphism, then $v$.inDegree ()$=\left(F_{\mathbb{V}}\right)_{/ v}$. inDegree () and $v$.outDegree ()$=\left(F_{\mathbb{V}}\right)_{v}$. outDegree () . The theorem is a consequence of $(92)$ and (90).

(95) If $F$ is isomorphism, then $v$.degree ()$=\left(F_{\mathbb{V}}\right)_{/ v}$.degree () . The theorem is a consequence of (89), (94), and (56). 


\section{InTo CHORD}

Now we state the proposition:

(96) Let us consider a graph $G$, and vertices $u, v, w$ of $G$. Suppose $v$ is endvertex and $u \neq w$. Then

(i) $u$ and $v$ are not adjacent, or

(ii) $v$ and $w$ are not adjacent.

Proof: Consider $e$ being an object such that $v$.edgesInOut ()$=\{e\}$ and $e$ does not join $v$ and $v$ in $G$. Consider $v^{\prime}$ being a vertex of $G$ such that $e$ joins $v$ and $v^{\prime}$ in $G$. Consider $e_{8}$ being an object such that $e_{8}$ joins $v$ and $u$ in $G$. There exists no object $e^{\prime}$ such that $e^{\prime}$ joins $v$ and $w$ in $G$.

Let us consider a graph $G$ and a vertex $v$ of $G$. Now we state the propositions:

(97) Suppose $3 \subseteq G$.order() and $v$ is endvertex. Then there exist vertices $u$, $w$ of $G$ such that

(i) $u \neq v$, and

(ii) $w \neq v$, and

(iii) $u \neq w$, and

(iv) $u$ and $v$ are adjacent, and

(v) $v$ and $w$ are not adjacent.

The theorem is a consequence of (96).

(98) Suppose $4 \subseteq G$.order() and $v$ is endvertex. Then there exist vertices $x$, $y, z$ of $G$ such that

(i) $v \neq x$, and

(ii) $v \neq y$, and

(iii) $v \neq z$, and

(iv) $x \neq y$, and

(v) $x \neq z$, and

(vi) $y \neq z$, and

(vii) $v$ and $x$ are adjacent, and

(viii) $v$ and $y$ are not adjacent, and

(ix) $v$ and $z$ are not adjacent.

The theorem is a consequence of (97), (17), and (96).

Let $G_{F}$ be a graph-yielding function. We say that $G_{F}$ is chordal if and only 
(Def. 4) for every object $x$ such that $x \in \operatorname{dom} G_{F}$ there exists a graph $G$ such that $G_{F}(x)=G$ and $G$ is chordal.

Let $G$ be a chordal graph. Let us note that $\langle G\rangle$ is chordal and $\mathbb{N} \longmapsto G$ is chordal.

Let $G_{F}$ be a non empty, graph-yielding function. Note that $G_{F}$ is chordal if and only if the condition (Def. 5) is satisfied.

(Def. 5) for every element $x$ of $\operatorname{dom} G_{F}, G_{F}(x)$ is chordal.

Let $G_{S q}$ be a graph sequence. Let us note that $G_{S q}$ is chordal if and only if the condition (Def. 6) is satisfied.

(Def. 6) for every natural number $n, G_{S q}(n)$ is chordal.

Let us observe that every graph-yielding function which is empty is also chordal and there exists a graph sequence which is chordal and there exists a graph-yielding finite sequence which is non empty and chordal.

Let $G_{F}$ be a chordal, non empty, graph-yielding function and $x$ be an element of $\operatorname{dom} G_{F}$. One can verify that $G_{F}(x)$ is chordal. Let $G_{S q}$ be a chordal graph sequence and $x$ be a natural number. One can verify that $G_{S q}(x)$ is chordal.

Let $p$ be a chordal, graph-yielding finite sequence and $n$ be a natural number. Note that $p\left\lceil n\right.$ is chordal and $p_{l n}$ is chordal. Let $m$ be a natural number. Let us observe that $\operatorname{smid}(p, m, n)$ is chordal and $\langle p(m), \ldots, p(n)\rangle$ is chordal.

Let $p, q$ be chordal, graph-yielding finite sequences. Note that $p^{\frown} q$ is chordal and $p \wedge q$ is chordal.

Let $G_{1}, G_{2}$ be chordal graphs. One can verify that $\left\langle G_{1}, G_{2}\right\rangle$ is chordal. Let $G_{3}$ be a chordal graph. One can check that $\left\langle G_{1}, G_{2}, G_{3}\right\rangle$ is chordal.

\section{INTO GLIB_011}

Now we state the propositions:

(99) Let us consider non-directed-multi graphs $G_{1}, G_{2}$, a directed partial vertex mapping $f$ from $G_{1}$ to $G_{2}$, and a vertex $v$ of $G_{1}$. Suppose $f$ is directed-isomorphism. Then

(i) $v$.inDegree ()$=f_{/ v}$.inDegree () , and

(ii) $v$.outDegree ()$=f_{/ v}$.outDegree () .

The theorem is a consequence of (94).

(100) Let us consider non-multi graphs $G_{1}, G_{2}$, a partial vertex mapping $f$ from $G_{1}$ to $G_{2}$, and a vertex $v$ of $G_{1}$. If $f$ is isomorphism, then $v$.degree ()$=$ $f_{/ v}$. degree(). The theorem is a consequence of (95). 


\section{REFERENCES}

[1] Grzegorz Bancerek, Czesław Byliński, Adam Grabowski, Artur Korniłowicz, Roman Matuszewski, Adam Naumowicz, Karol Pąk, and Josef Urban. Mizar: State-of-the-art and beyond. In Manfred Kerber, Jacques Carette, Cezary Kaliszyk, Florian Rabe, and Volker Sorge, editors, Intelligent Computer Mathematics, volume 9150 of Lecture Notes in Computer Science, pages 261-279. Springer International Publishing, 2015. ISBN 978-3319-20614-1. doi $10.1007 / 978-3-319-20615-8 \_17$.

[2] Grzegorz Bancerek, Czesław Byliński, Adam Grabowski, Artur Korniłowicz, Roman Matuszewski, Adam Naumowicz, and Karol Pak. The role of the Mizar Mathematical Library for interactive proof development in Mizar. Journal of Automated Reasoning, 61(1):9-32, 2018. do1 $10.1007 / \mathrm{s} 10817-017-9440-6$

[3] John Adrian Bondy and U. S. R. Murty. Graph Theory. Graduate Texts in Mathematics, 244. Springer, New York, 2008. ISBN 978-1-84628-969-9.

[4] Pavol Hell and Jaroslav Nesetril. Graphs and homomorphisms. Oxford Lecture Series in Mathematics and Its Applications; 28. Oxford University Press, Oxford, 2004. ISBN 0-19-852817-5.

[5] Ulrich Knauer. Algebraic graph theory: morphisms, monoids and matrices, volume 41 of De Gruyter Studies in Mathematics. Walter de Gruyter, 2011.

[6] Sebastian Koch. About graph mappings. Formalized Mathematics, 27(3):261-301, 2019. doi: $10.2478 /$ forma-2019-0024.

[7] Sebastian Koch. About graph complements. Formalized Mathematics, 28(1):41-63, 2020. doi:10.2478/forma-2020-0004.

[8] Gilbert Lee and Piotr Rudnicki. Alternative graph structures Formalized Mathematics, 13(2):235-252, 2005.

[9] Robin James Wilson. Introduction to Graph Theory. Oliver \& Boyd, Edinburgh, 1972. ISBN 0-05-002534-1.

Accepted December 30, 2019 